\title{
FIR Filter Design by Convex Optimization Using Directed Iterative Rank Refinement Algorithm
}

\author{
Mehmet Dedeoğlu, Student Member, IEEE, Yaşar Kemal Alp, and Orhan Arıkan, Member, IEEE
}

\begin{abstract}
The advances in convex optimization techniques have offered new formulations of design with improved control over the performance of FIR filters. By using lifting techniques, the design of a length- $L$ FIR filter can be formulated as a convex semidefinite program (SDP) in terms of an $L \times L$ matrix that must be rank-1. Although this formulation provides means for introducing highly flexible design constraints on the magnitude and phase responses of the filter, convex solvers implementing interior point methods almost never provide a rank-1 solution matrix. To obtain a rank-1 solution, we propose a novel Directed Iterative Rank Refinement (DIRR) algorithm, where at each iteration a matrix is obtained by solving a convex optimization problem. The semidefinite cost function of that convex optimization problem favors a solution matrix whose dominant singular vector is on a direction determined in the previous iterations. Analytically it is shown that the DIRR iterations provide monotonic improvement, and the global optimum is a fixed point of the iterations. Over a set of design examples it is illustrated that the DIRR requires only a few iterations to converge to an approximately rank-1 solution matrix. The effectiveness of the proposed method and its flexibility are also demonstrated for the cases where in addition to the magnitude constraints, the constraints on the phase and group delay of filter are placed on the designed filter.
\end{abstract}

Index Terms-Finite impulse response (FIR) filter design, spectral mask, convex optimization, semidefinite programming, semidefinite relaxation, iterative rank refinement.

\section{INTRODUCTION}

D IGITAL FINITE IMPULSE RESPONSE (FIR) filters have always been one of the prominent building blocks in digital signal processing because of their assured stability and efficient implementations based on the Fast Fourier Transform (FFT) [1]-[3]. A diverse class of FIR filter design techniques have been proposed in the literature including the ParksMcClellan algorithm [2], [4], optimization based techniques like METEOR [5] and peak-constrained least squares (PCLS)

Manuscript received May 29, 2015; revised November 05, 2015; accepted December 16, 2015. Date of publication January 06, 2016; date of current version March 29, 2016. The associate editor coordinating the review of this manuscript and approving it for publication was Prof. Shuguang Cui.

M. Dedeoğlu was with the Department of Electrical and Electronics Engineering, Bilkent University, Ankara 06800, Turkey. He is now with the School of Electrical, Computer, and Energy Engineering, Arizona State University, Tempe, AZ 85287 USA (e-mail: mdedeogl@asu.edu)

Y. K. Alp was with the Department of Electrical and Electronics Engineering of Bilkent University, Ankara 06800, Turkey. He is now with the Radar, Electronic Warfare, and Intelligence Systems Division, ASELSAN Inc., Ankara, Turkey (e-mail: ykalp@aselsan.com.tr).

O. Arrkan is with the Department of Electrical and Electronics Engineering, Bilkent University, Ankara 06800, Turkey (e-mail: oarikan@ee.bilkent.edu.tr).

Color versions of one or more of the figures in this paper are available online at http://ieeexplore.iee.org.

Digital Object Identifier 10.1109/TSP.2016.2515062
[6], [7], and Chebyshev approximation based methods [8]-[11]. Typically, techniques that are based on optimization are slower, but capable of obtaining optimal solutions that satisfy a set of design constraints.

With the development of the convex solvers implementing fast interior point methods, new, optimization based FIR filter design approaches based on convex modelling have emerged [12]-[24]. In [12] and [14], the non-linear phase FIR filter design problem is modeled as a linear program (LP), where the variables of optimization are the deterministic autocorrelation sequence of the filter coefficients which are obtained by applying spectral factorization to the solution of the constructed LP. This framework is extended to linear phase FIR filter design by using discrete cosine transform (DCT) instead of discrete Fourier transform (DFT) in [12]. However, since the LP formulation operates on the autocorrelation sequence of the filter coefficients, the incorporation of additional constraints on the phase response of the filter is not possible. A second order cone programming (SOCP) formulation of FIR filter design that is based on the weighted least-squares approach is proposed in [15]. Although this technique can constrain the deviation from a prototype group delay response, its convergence is highly dependent on the initial choice of the prototype filter because of the nonconvexity of the constructed SOCP. In [17], another constrained least-squares based design that can implement the phase and group delay error constraints, is presented. Since the constructed optimization problem is a semi-infinite positive-definite formulation, the optimization problem is solved using the GoldfarbIdnani algorithm. Though the underlying Goldfarb-Idnani algorithm converges in a few iterations, the obtained solutions do not possess optimality. In addition, the convexity of the proposed formulation depends on the positive-definiteness of a constructed Hessian matrix. In [18], a more general, non-convex filter design problem is cast as a convex problem and solved using a Goldfarb-Idnani based algorithm. The constructed iterative optimization problem does not require any phase response specifications. However, the optimality of the original $L_{p}$-constrained error minimization problem is not guaranteed.

A semidefinite programming based FIR filter design technique, which makes use of spectral mask constraints, is proposed in [23]. Since the semidefinite program (SDP) is constructed using linear matrix inequalities (LMI), convex spectral mask constraints such as magnitude mask constraints, can be implemented efficiently without using frequency sampling. However, the phase and group delay constraints cannot be implemented in LMIs since quadratic representations of these constraints typically possess indefinite constraint matrices. A more general formulation, in which the objective and the quadratic constraints 
form an SDP, is proposed in [24]. In this formulation, the variables of optimization are restricted to form a rank-1, positive semidefinite (PSD) matrix. However, the convex solvers implementing interior point methods almost never provide rank-1 solution matrices for SDPs [24]. A commonly used approach to obtain the filter coefficients is to decompose the rank-1 approximation of the solution matrix to obtain the filter coefficients. Unfortunately, this generates a filter, which typically violates the design constraints.

In this work, we propose a highly flexible FIR filter design technique that is based on semidefinite programming. First, we construct a non-convex quadratically constrained quadratic program (QCQP), where both constraints on the magnitude and phase responses of the filter can be incorporated. Then, by relaxing the constructed QCQP, a convex SDP is obtained and its global optimum is found by utilizing a convex solver, such as YALMIP [25], SeDuMi [26] or SDPT3 [27]. To establish convergence to a rank-1 solution matrix, we propose the novel Directed Iterative Rank Refinement (DIRR) algorithm, in which the energy of the singular value corresponding to a particular singular vector can be increased at each iteration. For improved convergence, the energy of the singular value associated with the closest singular vector to the singular vector chosen in the previous iteration is increased with respect to the total energy in the remaining singular values. As it will be detailed in Section-3, the DIRR iterations provide monotonic improvement. Since the cost is bounded from below, convergence of its iterations are guaranteed. However, the iterations may converge to a local minimum. If however, the iterations are started with the global optimum solution, the iterations will not move away from this solution, i.e., the global optimum is fixed point of iterations. Finally, if there is no local minimum other than the global minimum, the iterations will converge to the global minimum. The effectiveness of the proposed method and its flexibility are also demonstrated on cases where in addition to the magnitude constraints, the constraints on the phase and group delay of the filter are placed on the designed filter.

The outline of the presentation is as follows; in Section 2, the preliminaries on convex-optimization-based FIR filter design are given. In Section 3, the proposed DIRR technique is introduced and its convergence properties are investigated. Design examples for various filter specifications and the comparisons with the results obtained by using alternative approaches are provided in Section 4. Finally, the concluding remarks are given in Section 5. Throughout the paper, bold lower case and bold capital letters represent vectors and matrices; $(\cdot)^{H}$ and $(\cdot)^{T}$ are hermitian and transpose operations; $\operatorname{Re}(\cdot)$ and $\operatorname{Im}(\cdot)$ denote the real and the imaginary parts of their arguments, respectively.

\section{BRIEF REVIEW OF OptimizAtion BASED FIR FILTER DESIGN}

An FIR filter of length $L$ is typically characterized by either its impulse response $h_{n}, 0 \leq n \leq L-1$, or its frequency response

$$
H(\omega)=\sum_{n=0}^{L-1} h_{n} e^{-j \omega n}, \forall \omega \in[-\pi, \pi] .
$$

In optimization based FIR filter design, the filter coefficients are typically obtained by imposing a set of desired constraints on the

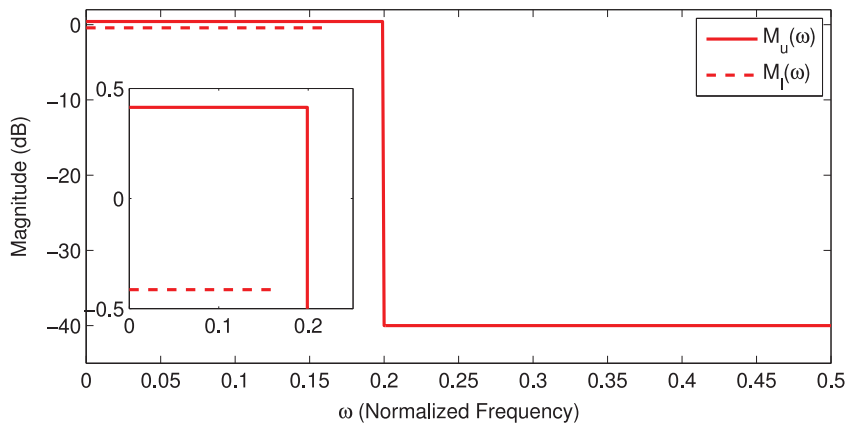

Fig. 1. A typical set of mask constraints on the magnitude response of an FIR filter. $M_{u}(\omega)$ and $M_{l}(\omega)=1 / 1.1$ are the upper and lower masks with transition band $\omega_{p}=0.16 \leq \omega \leq 0.20=\omega_{s}$, respectively. $M_{u}(\omega)=1.1$ in the pass band and $M_{u}(\omega)=0.0001$ in the stop band.

frequency response of the filter as in the following feasibility problem:

$$
\begin{aligned}
& \text { find } h_{n} \in \mathbb{R}, 0 \leq n \leq L-1 \\
& \text { s.t. }|H(\omega)|^{2} \leq M_{u}(\omega), \forall \omega \in[0, \pi], \\
&|H(\omega)|^{2} \geq M_{l}(\omega), \forall \omega \in\left[0, \omega_{p}\right],
\end{aligned}
$$

where, as shown in Fig. 1, $\omega_{p}$ is the largest frequency in the passband, $M_{u}(\omega)$ and $M_{l}(\omega)$ are the upper and lower mask constraints on the magnitude response, respectively. The feasibility problem given in (2) can be expressed in vector form as:

$$
\begin{aligned}
& \text { find } \mathbf{h} \in \mathbb{R}^{L} \\
& \text { s.t. } \mathbf{h}^{T} \mathbf{A}(\omega) \mathbf{h} \leq M_{u}(\omega), \forall \omega \in[0, \pi] \\
& \mathbf{h}^{T} \mathbf{A}(\omega) \mathbf{h} \geq M_{l}(\omega), \forall \omega \in\left[0, \omega_{p}\right] .
\end{aligned}
$$

Here, $\mathbf{h}=\left[h_{0}, h_{1}, \ldots, h_{L-1}\right]^{T}$ and $\mathbf{A}(\omega)=\mathbf{v}(\omega) \mathbf{v}^{H}(\omega)$, where $\mathbf{v}(\omega)=\left[1, e^{j \omega}, \ldots, e^{j \omega(L-1)}\right]^{T}$. Since $\omega$ is defined in an interval, this feasibility problem has uncountably many constraints. In practice, by using a dense enough frequency domain sampling, a quadratic feasibility problem with finite number of constraints can be obtained as:

$$
\begin{array}{ll}
\text { find } \mathbf{h} \in \mathbb{R}^{L} & \\
\text { s.t. } & \mathbf{h}^{T} \mathbf{A}_{k} \mathbf{h} \leq a_{k}, \quad 0 \leq k \leq K_{u}, \\
& \mathbf{h}^{T} \mathbf{A}_{k} \mathbf{h} \geq b_{k}, \quad 0 \leq k \leq K_{l},
\end{array}
$$

where $\mathbf{A}_{k}=\mathbf{v}\left(\omega_{k}\right) \mathbf{v}\left(\omega_{k}\right)^{H}, a_{k}=M_{u}\left(\omega_{k}\right)$ and $b_{k}=M_{l}\left(\omega_{k}\right)$. For a uniform frequency spacing of $\pi / K$, where $K$ is the number of the frequency samples, the resulting numbers of the upper and lower frequency mask constraints are $K_{u}=K$ and $K_{l}=\left\lceil K w_{p} / \pi\right\rceil$. Here, $\lceil\cdot\rceil$ indicates the smallest integer that is greater than its argument. A rule of thumb for selecting $K$ is $K$ $\approx 15 L$ [2]. However, as investigated in [28]-[30], judiciously chosen set of non-uniformly spaced frequency samples provides acceptable designs requiring fewer number of constraints.

In addition to the spectral mask constraints, minimization of the stop band energy can be achieved as in the following formulation:

$$
\begin{aligned}
\min _{\mathbf{h} \in \mathbb{R}^{L}} & \mathbf{h}^{T} \mathbf{A}_{s} \mathbf{h} \\
\text { s.t. } & \mathbf{h}^{T} \mathbf{A}_{k} \mathbf{h} \leq a_{k}, \quad 0 \leq k \leq K_{u}, \\
& \mathbf{h}^{T} \mathbf{A}_{k} \mathbf{h} \geq b_{k}, \quad 0 \leq k \leq K_{l} .
\end{aligned}
$$

Here $\mathbf{A}_{s}=\int_{\omega_{s}}^{\pi} W(\omega) \mathbf{A}(\omega) d \omega$, where $W(\omega) \geq 0$ is a weighting function providing further flexibility and control on 
the design. The optimization problem in (5) has a quadratic cost and a set of quadratic constraints, hence it is a QCQP [31].

In general, the filter coefficients obtained as a solution to (5) has non-linear phase response [12], [23], [32]. Filters with linear phase responses can also be obtained by constraining filter coefficients to be symmetric in (5). For instance, to obtain a real, type-1 FIR filter, the following constraints should be incorporated to (5) [2]:

$$
h_{n}=h_{L-1-n}, \quad 0 \leq n \leq(L-1) / 2 .
$$

These constraints can be equivalently expressed as:

$$
\mathbf{h}=\mathbf{\Pi} \boldsymbol{\theta}
$$

where, $\boldsymbol{\theta}=\left[h_{0}, h_{1}, \ldots, h_{(L-1) / 2}\right]^{T}$ is the vector of reduced filter coefficients, and $\mathbf{\Pi}=\left[\mathbf{I}^{T}, \hat{\mathbf{I}}^{T}\right]^{T}$ with $\mathbf{I}$ is the identity matrix of size $(L+1) / 2 \times(L+1) / 2$ and $\hat{\mathbf{I}}$ is the flipping matrix of size $(L-1) / 2 \times(L+1) / 2$ composed of zeros except for the entries of $\left(i, \frac{L+1}{2}-i\right), 1 \leq i \leq \frac{L-1}{2}$ that are equal to 1 . Hence, the optimization problem stated in (5) for designing type-1 FIR filters of odd length $L$ takes the following form:

$$
\begin{aligned}
& \min _{\boldsymbol{\theta} \in \mathbb{R}^{(L+1) / 2}} \boldsymbol{\theta}^{T} \boldsymbol{\Pi}^{T} \mathbf{A}_{s} \boldsymbol{\Pi} \boldsymbol{\theta} \\
& \text { s.t. } \boldsymbol{\theta}^{T} \boldsymbol{\Pi}^{T} \mathbf{A}_{k} \boldsymbol{\Pi} \boldsymbol{\theta} \leq a_{k}, \quad 0 \leq k \leq K_{u}, \\
& \boldsymbol{\theta}^{T} \boldsymbol{\Pi}^{T} \mathbf{A}_{k} \boldsymbol{\Pi} \boldsymbol{\theta} \geq b_{k}, \quad 0 \leq k \leq K_{l} .
\end{aligned}
$$

Quadratic inequality constraints of the form $\mathbf{x}^{T} \mathbf{W} \mathbf{x} \geq y$ with a PSD matrix $\mathbf{W}$ do not define convex sets [33]. Note that $\boldsymbol{\Pi}^{T} \mathbf{A}_{k} \boldsymbol{\Pi}$ is a PSD matrix for $0 \leq k \leq K_{u}$. Hence, the constructed QCQPs with quadratic lower mask constraints are not convex as well. Therefore, efficient convex solvers cannot be used directly to obtain their solutions. In the next section, by using semidefinite formulation techniques, the QCQP stated in this section will be convexified. In addition, constraints on the phase and group delay will be incorporated to the design problem.

\section{Proposed FIR Filter Design by Semidefinite PROGRAMMING}

In the QCQP formulation of FIR filter design given in (5), the filter coefficients are chosen to minimize the stop band energy subject to the magnitude constraints. Since the quadratic constraints $\mathbf{h}^{T} \mathbf{A}_{k} \mathbf{h} \geq b_{k}$ define non-convex sets, the resulting optimization problem is also non-convex. However, it can be relaxed to a convex optimization problem by using the lifting technique given in [24]. For this purpose, first (5) is equivalently expressed as:

$$
\begin{aligned}
\min _{\mathbf{h} \in \mathbb{R}^{L}} & \operatorname{Tr}\left(\mathbf{A}_{s} \mathbf{h} \mathbf{h}^{T}\right) \\
\text { s.t. } & \operatorname{Tr}\left(\mathbf{A}_{k} \mathbf{h} \mathbf{h}^{T}\right) \leq a_{k}, 0 \leq k \leq K_{u}, \\
& \operatorname{Tr}\left(\mathbf{A}_{k} \mathbf{h h}^{T}\right) \geq b_{k}, 0 \leq k \leq K_{l},
\end{aligned}
$$

where $\operatorname{Tr}(\cdot)$ is the trace operator. By lifting the problem into matrix space [24], [34], $\mathbf{H}=\mathbf{h h}^{T}$ can be defined as the variable of the optimization with the introduction of two additional constraints on its positive definiteness and rank:

$$
\begin{aligned}
\min _{\mathbf{H} \in \mathbb{R}^{L \times L}} & \operatorname{Tr}\left(\mathbf{A}_{s} \mathbf{H}\right) \\
\text { s.t. } & \operatorname{Tr}\left(\mathbf{A}_{k} \mathbf{H}\right) \leq a_{k}, 0 \leq k \leq K_{u}, \\
& \operatorname{Tr}\left(\mathbf{A}_{k} \mathbf{H}\right) \geq b_{k}, 0 \leq k \leq K_{l}, \\
& \mathbf{H} \succeq 0 \\
& \operatorname{rank}(\mathbf{H})=1,
\end{aligned}
$$

which is an SDP in $\mathbb{R}^{L \times L}$. Although the lifted formulation in (10) has $L(L+1) / 2$ unknowns compared to the $L$ unknowns in (5), its constraints have become linear in terms of the entries of $\mathbf{H}$. Note that, the third and fourth constraints in (10) guarantee that the matrix $\mathbf{H}$ has a real valued decomposition $\mathbf{H}=\mathbf{h h}^{T}$ [24].

Since the optimization problems given in (5) and (10) are equivalent, if there exist an optimal solution $\mathbf{h}^{\star}$ for (5), then $\mathbf{H}^{\star}=\mathbf{h}^{\star} \mathbf{h}^{\star T}$ is an optimal solution to (10). Conversely, if $\mathbf{H}^{\star}$ is the optimal solution of (10), it can be factored as $\mathbf{H}^{\star}=\mathbf{h}^{\star} \mathbf{h}^{\star T}$, where $\mathbf{h}^{\star}$ is an optimal solution to (5). However, due to the rank-1 constraints on $\mathbf{H},(10)$ is still a non-convex optimization problem. By removing the rank-1 constraint, (10) can be relaxed to obtain a convex SDP as in [24], [33], [35]:

$$
\begin{aligned}
\min _{\mathbf{H} \in \mathbb{R}^{L \times L}} & \operatorname{Tr}\left(\mathbf{A}_{s} \mathbf{H}\right) \\
\text { s.t. } & \operatorname{Tr}\left(\mathbf{A}_{k} \mathbf{H}\right) \leq a_{k}, 0 \leq k \leq K_{u}, \\
& \operatorname{Tr}\left(\mathbf{A}_{k} \mathbf{H}\right) \geq b_{k}, 0 \leq k \leq K_{l}, \\
& \mathbf{H} \succeq 0 .
\end{aligned}
$$

The relaxed SDP in (11) can be solved by utilizing the SDP solvers such as SeDuMi [26] or SDPT3 [27]. However, the global optimizer $\mathbf{H}^{*}$ of (11) can significantly deviate from a rank-1 matrix. Therefore, the optimal filter coefficients that can be approximated as:

$$
\tilde{\mathbf{h}}^{*}=\sqrt{\sigma_{1}^{*}} \mathbf{u}_{1}^{*},
$$

where $\sigma_{1}^{*}$ is the largest singular value of the $\mathbf{H}^{*}$ and $\mathbf{u}_{1}^{*}$ is the corresponding singular vector, typically provides a poor approximation to the actual $\mathbf{h}^{\star}$, and violates the constraints in (5). Even though there exist low rank matrix factorization techniques for SDPs [3], [36]-[38], these techniques cannot be used to obtain a rank-1 solution matrix for SDPs under a high number of affine constraints as in (10). Although not detailed here, the high number of affine constraints as in (10) create significant challenges for the randomization based approaches as well [39]. To overcome this difficulty, we propose a novel iterative algorithm, where the cost function of the constructed SDP is updated at each iteration to enforce the iterations towards a rank-1 solution matrix. The proposed algorithm makes use of the singular value decomposition (SVD) of the obtained matrix $\mathbf{H}^{i}$ at iteration $i$ to formulate an updated SDP to be solved in the next iteration. Let $\mathbf{H}^{i}$ be a feasible point of the following feasibility problem:

$$
\begin{aligned}
\text { find } & \mathbf{H}^{i} \in \mathbb{R}^{L \times L} \\
\text { s.t. } & \operatorname{Tr}\left(\mathbf{A}_{k} \mathbf{H}^{i}\right) \leq a_{k}, 0 \leq k \leq K_{u}, \\
& \operatorname{Tr}\left(\mathbf{A}_{k} \mathbf{H}^{i}\right) \geq b_{k}, 0 \leq k \leq K_{l}, \\
& \mathbf{H}^{i} \succeq 0 .
\end{aligned}
$$

Since $\mathbf{H}^{i}$ is a PSD and symmetric matrix, its singular value decomposition is in the following form:

$$
\mathbf{H}^{i}=\mathbf{U}^{i} \boldsymbol{\Sigma}^{i} \mathbf{U}^{i^{T}}=\sum_{l=1}^{L} \sigma_{l}^{i} \mathbf{u}_{l}^{i} \mathbf{u}_{l}^{i^{T}} .
$$

Here, $\mathbf{U}^{i}=\left[\mathbf{u}_{1}^{i}, \mathbf{u}_{2}^{i}, \ldots, \mathbf{u}_{L}^{i}\right]$ where $\mathbf{u}_{l}^{i}, 1 \leq l \leq L$ are the singular vectors and $\boldsymbol{\Sigma}^{i}=\operatorname{diag}\left(\left[\sigma_{1}^{i}, \ldots, \sigma_{L}^{i}\right]\right)$, where $\sigma_{1}^{i} \geq \cdots \geq$ $\sigma_{L}^{i} \geq 0$ are the ordered singular values of $\mathbf{H}^{i}$. Since the energy of $\mathbf{H}^{i}$ is upper bounded by the upper mask constraints $M_{u}(\omega)$, 
maximizing the energy of $\mathbf{H}^{i+1}$ along the $\ell$ th singular vector $\mathbf{u}_{\ell}^{i}$ of $\mathbf{H}^{i}$ in the next iteration will be a bounded objective. The maximization of the energy of $\mathbf{H}^{i+1}$ along the direction of $\mathbf{u}_{\ell}^{i}$ can be added to (13) as a cost function in the updated SDP form as:

$$
\begin{aligned}
\min _{\mathbf{H}^{i+1} \in \mathbb{R}^{L \times L}}- & \operatorname{Tr}\left(\mathbf{u}_{\ell}^{i} \mathbf{u}_{\ell}^{i^{T}} \mathbf{H}^{i+1}\right) \\
\text { s.t. } & \operatorname{Tr}\left(\mathbf{A}_{k} \mathbf{H}^{i+1}\right) \leq a_{k}, \quad 0 \leq k \leq K_{u}, \\
& \operatorname{Tr}\left(\mathbf{A}_{k} \mathbf{H}^{i+1}\right) \geq b_{k}, \quad 0 \leq k \leq K_{l}, \\
& \mathbf{H}^{i+1} \succeq 0 .
\end{aligned}
$$

If in the iterations, the preferred direction is chosen as the dominant singular vector of the previous iteration, i.e., $\ell=1$, the following facts can be proven for the iterations in (15).

Proposition 1: The cost $J^{i}\left(\mathbf{H}^{i}\right)=-\operatorname{Tr}\left(\mathbf{u}_{1}^{i-1}\left(\mathbf{u}_{1}^{i-1}\right)^{T} \mathbf{H}^{i}\right)$ of the updated constructed SDP that is given in (15) monotonically decreases at each iteration:

$$
J^{i+1}\left(\mathbf{H}^{i+1}\right) \leq J^{i}\left(\mathbf{H}^{i}\right),
$$

where $\mathbf{H}^{i}$ and $\mathbf{H}^{i+1}$ are the optimal solutions obtained in iterations $i$ and $i+1$, respectively.

Proof: Since the feasible set of the optimization problem remains the same for all iterations,

$$
J^{i+1}\left(\mathbf{H}^{i+1}\right) \leq J^{i+1}\left(\mathbf{H}^{i}\right)
$$

always holds. As given in (14), $\mathbf{H}^{i}$ has the following singular value decomposition:

$$
\mathbf{H}^{i}=\sum_{l=1}^{L} \sigma_{l}^{i} \mathbf{u}_{l}^{i}\left(\mathbf{u}_{l}^{i}\right)^{T}
$$

with $\sigma_{1}^{i} \geq \sigma_{2}^{i} \geq \cdots \geq \sigma_{L}^{i} \geq 0$ and $\left\|\mathbf{u}_{l}^{i}\right\|_{2}=1, \forall l$. Hence,

$$
\begin{aligned}
J^{i+1}\left(\mathbf{H}^{i}\right) & =-\operatorname{Tr}\left(\mathbf{u}_{1}^{i}\left(\mathbf{u}_{1}^{i}\right)^{T} \mathbf{H}^{i}\right) \\
& =-\left(\mathbf{u}_{1}^{i}\right)^{T} \mathbf{H}^{i} \mathbf{u}_{1}^{i} \\
& =-\sigma_{1}^{i} .
\end{aligned}
$$

Similarly, the cost function evaluated at $\mathbf{H}^{i}$ at iteration $i$ can be written as:

$$
\begin{aligned}
J^{i}\left(\mathbf{H}^{i}\right) & =-\operatorname{Tr}\left(\mathbf{u}_{1}^{i-1}\left(\mathbf{u}_{1}^{i-1}\right)^{T} \mathbf{H}^{i}\right) \\
& =-\left(\mathbf{u}_{1}^{i-1}\right)^{T} \mathbf{H}^{i} \mathbf{u}_{1}^{i-1} \\
& \geq \min _{\|\mathbf{v}\|_{2}=1}-\mathbf{v}^{T} \mathbf{H}^{i} \mathbf{v} \\
& =-\sigma_{1}^{i}=J^{i+1}\left(\mathbf{H}^{i}\right),
\end{aligned}
$$

with equality if and only if $\mathbf{H}^{i}=\mathbf{H}^{i-1}$. Hence,

$$
J^{i+1}\left(\mathbf{H}^{i}\right) \leq J^{i}\left(\mathbf{H}^{i}\right) .
$$

Then, together with (17), the claim in (16) follows.

Next, we define a non-convex optimization problem whose minimum provides a lower bound to the cost function of (15).

Proposition 2: The cost function of (15) is lower bounded by the global optimum value of the following non-convex optimization problem:

$$
\begin{aligned}
\min _{\mathbf{H} \in \mathbb{R}^{L \times L}}- & \operatorname{Tr}(\mathbf{H}) \\
\text { s.t. } & \operatorname{Tr}\left(\mathbf{A}_{k} \mathbf{H}\right) \leq a_{k}, \quad 0 \leq k \leq K_{u} \\
& \operatorname{Tr}\left(\mathbf{A}_{k} \mathbf{H}\right) \geq b_{k}, \quad 0 \leq k \leq K_{l} \\
& \mathbf{H} \succeq 0, \\
& \operatorname{rank}(\mathbf{H})=1 .
\end{aligned}
$$
by:

Proof: Consider the relaxed convex version of (22) given

$$
\begin{aligned}
\min _{\mathbf{H} \in \mathbb{R}^{L \times L}}-\operatorname{Tr}(\mathbf{H}) & \\
\text { s.t. } & \operatorname{Tr}\left(\mathbf{A}_{k} \mathbf{H}\right) \leq a_{k}, \quad 0 \leq k \leq K_{u}, \\
& \operatorname{Tr}\left(\mathbf{A}_{k} \mathbf{H}\right) \geq b_{k}, \quad 0 \leq k \leq K_{l}, \\
& \mathbf{H} \succeq 0,
\end{aligned}
$$

where the non-convex $\operatorname{rank}(\mathbf{H})=1$ constraint in $(22)$ is removed. Let $\overline{\mathbf{H}}^{\star}$ be the optimal solution of (23), which is not necessarily rank-1, with its corresponding cost $-\operatorname{Tr}\left(\overline{\mathbf{H}}^{\star}\right)=$ $-\sum_{i=1}^{L} \bar{\sigma}_{i}^{\star}$, where $\bar{\sigma}_{i}^{\star}, i=1,2, \ldots, L$ are the singular values of $\overline{\mathbf{H}}^{\star}$. Note that $-\operatorname{Tr}\left(\overline{\mathbf{H}}^{\star}\right) \leq-\operatorname{Tr}\left(\mathbf{H}^{i}\right) \leq-\sigma_{1}^{i}=J^{i+1}\left(\mathbf{H}^{i}\right) \leq$ $J^{i}\left(\mathbf{H}^{i}\right)$ always holds because of (20) and the equivalence of the feasible sets of (15) and (23). Then, because of the coexistence of rank-1 and higher rank solutions, which is shown in Section 4.1.1.3 of [35], there exists a rank-1 solution matrix with exactly the same cost $-\operatorname{Tr}\left(\overline{\mathbf{H}}^{\star}\right)=-\operatorname{Tr}\left(\hat{\mathbf{H}}^{\star}\right)$, where $\hat{\mathbf{H}}^{\star}$ is the optimal solution matrix of (22). Hence, the cost function of (15) is lower bounded by the global optimum value of (22):

$$
-\operatorname{Tr}\left(\hat{\mathbf{H}}^{\star}\right) \leq J^{i}\left(\mathbf{H}^{i}\right), \forall i .
$$

The coexistence of rank-1 and higher rank solutions can be visualized in the following way. Since $\overline{\mathbf{H}}^{\star}$ is PSD and symmetric, $\overline{\mathbf{H}}^{\star}$ can uniquely be transformed to an autocorrelation sequence $\overline{\mathbf{r}}^{\star}$ as:

$$
\begin{aligned}
\overline{\mathbf{r}}_{k}^{\star}=\sum_{m=\max (1,1+k)}^{\min (L, L+k)} \overline{\mathbf{H}}^{\star}(m-k, m), \\
\quad \text { for }-L+1 \leq k \leq L-1,
\end{aligned}
$$

where $\overline{\mathbf{r}}_{k}^{\star}$ denotes the $k$ th entry of the vector $\overline{\mathbf{r}}^{\star}$ [35]. Then, the filter coefficients $\overline{\mathbf{x}}^{\star}$ can be obtained by decomposing $\overline{\mathbf{r}}^{\star}$ via spectral factorization [35]. Hence, a rank-1 matrix $\overline{\mathbf{x}}^{\star}\left(\overline{\mathbf{x}}^{\star}\right)^{T}$ yields the same objective value $-\operatorname{Tr}\left(\overline{\mathbf{H}}^{\star}\right)$.

Next, we prove that the optimal solution of (22) is a fixed point of the iterations in (15).

Proposition 3: $\hat{\mathbf{H}}^{\star}$, the optimal solution to (22), can be decomposed as:

$$
\hat{\mathbf{H}}^{\star}=\hat{\sigma}_{1}^{\star}\left(\hat{\mathbf{u}}_{1}^{\star}\right)^{T} \hat{\mathbf{u}}_{1}^{\star},
$$

where $\hat{\sigma}_{1}^{\star}>0$ and $\left\|\hat{\mathbf{u}}_{1}^{\star}\right\|_{2}=1$, is a fixed point of iterations in (15).

Proof: The coexistence property dictates that, if a high rank solution exists in the feasible set of (23), then a rank-1 solution also exists in the same feasible set [35]. Since the feasible sets of (23) and (15) are identical, $\hat{\mathbf{H}}^{\star}$, which possesses the largest singular value $\hat{\sigma}_{1}^{\star}$ in the feasible set, is also a candidate solution for (15). Hence, if $\hat{\mathbf{H}}^{\star}$, the optimal solution of (22), can be reached by the proposed DIRR formulation at iteration $i$, then the optimization problem at the $(i+1)$ th iteration becomes:

$$
\begin{aligned}
\min _{\mathbf{H}^{i+1} \in \mathbb{R}^{L \times L}}- & \operatorname{Tr}\left(\hat{\mathbf{u}}_{1}^{\star}\left(\hat{\mathbf{u}}_{1}^{\star}\right)^{T} \mathbf{H}^{i+1}\right) \\
\text { s.t. } & \operatorname{Tr}\left(\mathbf{A}_{k} \mathbf{H}^{i+1}\right) \leq a_{k}, 0 \leq k \leq K_{u}, \\
& \operatorname{Tr}\left(\mathbf{A}_{k} \mathbf{H}^{i+1}\right) \geq b_{k}, 0 \leq k \leq K_{l}, \\
& \mathbf{H}^{i+1} \succeq 0,
\end{aligned}
$$


where $\hat{\mathbf{H}}^{\star}=\hat{\sigma}_{1}^{\star} \hat{\mathbf{u}}_{1}^{\star}\left(\hat{\mathbf{u}}_{1}^{\star}\right)^{T}$ as $\hat{\mathbf{H}}^{\star}$ is rank-1. Because of (17), (19) and (24), the cost function of (27) can be lower and upper bounded as:

$$
-\hat{\sigma}_{1}^{\star} \leq-\operatorname{Tr}\left(\hat{\mathbf{u}}_{1}^{\star}\left(\hat{\mathbf{u}}_{1}^{\star}\right)^{T} \mathbf{H}^{i+1}\right) \leq J^{i+1}\left(\hat{\mathbf{H}}^{\star}\right)=-\hat{\sigma}_{1}^{\star},
$$

which is attained only for the choice of $\mathbf{H}^{i+1}=\hat{\mathbf{H}}^{\star}=\mathbf{H}^{i}$. Hence, if $\hat{\mathbf{H}}^{\star}$ is the optimal solution to (22), then it is a fixed point of the proposed iterations in (15).

As Proposition 1 suggests, the cost function of (15) monotonically decreases and the gap $\mu$ between $-\left(\mathbf{u}_{1}^{i-1}\right)^{T} \mathbf{H}^{i} \mathbf{u}_{1}^{i-1}$ and $\min _{\mathbf{H}^{i}}\left\{-\left(\mathbf{u}_{1}^{i-1}\right)^{T} \mathbf{H}^{i} \mathbf{u}_{1}^{i-1}\right\}$ is strictly positive, that is $\mu=$ $\sum_{k=1}^{L}\left(\sigma_{1}^{i}-\sigma_{k}^{i}\right)\left(\left(\mathbf{u}_{1}^{i-1}\right)^{T} \mathbf{u}_{k}^{i}\right)^{2}>0$ if $\left\langle\mathbf{u}_{1}^{i-1}, \mathbf{u}_{k}^{i}\right\rangle \neq 0$ for $\sigma_{1}^{i}>\sigma_{k}^{i}$ and $k \in[2, L]$. On the other hand, if the gap is zero, then the singular vectors of solutions at the current and previous iterations are the same and the inner product $\left\langle\mathbf{u}_{1}^{i-1}, \mathbf{u}_{k}^{i}\right\rangle=0$, for $\sigma_{1}^{i}>\sigma_{k}^{i}$ and $k \in[2, L]$. This implies the convergence of the iterations to a local minimum that can be identified by computing the following ratio:

$$
R^{i}=\sigma_{1}^{i} /\left(\sum_{l=1}^{L} \sigma_{l}^{i}\right),
$$

and comparing it with a threshold $\rho$, that is typically chosen as $\rho=1-10^{-4}$. If $\rho$ is exceeded by $R^{i}$, then an almost rank- 1 solution is obtained by the DIRR and the iterations are terminated. On the other hand, if $R^{i}<\rho$, then $\ell$ is incremented by one and the DIRR iterations that are given in Algorithm 1 and Algorithm 2 are continued. Note that, even if $\ell$ takes an arbitrary value in $[1, L]$, the iterations might still converge to a local minima. In cases, at which a rank-1 solution could not be found, various $a d$-hoc procedures such as constraining a set of singular values $\sigma_{l}$ corresponding to the singular vectors $\mathbf{u}_{l}$ for $l_{l} \leq l \leq l_{u}$ by a user defined upper limit $\nu$ or minimizing the negative energy in multiple directions simultaneously rather than minimizing the cost only on the $\ell$ th direction, can also be applied at the expense of computational complexity.

\section{Algorithm 1: The DIRR algorithm:}

1: $\mathbf{u}_{\ell}^{i=0} \mathbf{u}_{\ell}^{i=0^{T}} \leftarrow \mathbf{0}$.

2: Solve (30), (31), (40), (54) 1 .

3: if Optimization problem is feasible then

4: Apply SVD to $\mathbf{H}^{i=1}$

5: $\quad$ if $R_{j=0}^{1} \geq \rho$ in (29) then

6: Optimization is successful: $\mathbf{H}^{I} \leftarrow \mathbf{H}^{i=1}$.

7: else

8: $\quad j \leftarrow 1$.

9: $\quad$ while $j \leq L$ and $R_{j-1}^{i} \leq \rho$ do

10: Apply Algorithm 2 with $\ell=j$.

11: $\quad j \leftarrow j+1$.

12: end while

13: end if

14: Apply SVD to $\mathbf{H}^{I}$, and find its singular values.

15: $\mathbf{h}=\mathbf{u}_{1}^{I} \sqrt{\sigma_{1}^{I}}$.

16: end if

1(30), (31), (40) and (54) are solved for Design 1, 2; Design 3, Design 4 and Design 5, respectively.

\section{Algorithm 2}

1: $i \leftarrow 1, R^{i=1} \leftarrow R_{j-1}^{i}$.

2: while $R^{i} \leq \rho$ and $i \leq N_{\text {iter }}$ do

3: $i \leftarrow i+1$.

4: Solve (30), (31), (40), (54)1.

5: Apply SVD to $\mathbf{H}^{i}$.

6: Find $R^{i}$ using (29) and update $\lambda\left(R^{i}\right)$.

7: end while

8: $R_{j}^{i} \leftarrow R^{i}, \mathbf{H}^{I} \leftarrow \mathbf{H}^{i}$.

\section{A. Design With Magnitude Response Constraints}

To achieve a minimum stop band energy with a rank-1 solution matrix, at each iteration the following SDP is solved:

$$
\begin{aligned}
\min _{\mathbf{H}^{i+1} \in \mathbb{R}^{L \times L}} & \operatorname{Tr}\left(\left((1-\lambda) \mathbf{A}_{s}-\lambda \mathbf{u}_{\ell}^{i} \mathbf{u}_{\ell}^{i T}\right) \mathbf{H}^{i+1}\right) \\
\text { s.t. } & \operatorname{Tr}\left(\mathbf{A}_{k} \mathbf{H}^{i+1}\right) \leq a_{k}, \quad 0 \leq k \leq K_{u}, \\
& \operatorname{Tr}\left(\mathbf{A}_{k} \mathbf{H}^{i+1}\right) \geq b_{k}, \quad 0 \leq k \leq K_{l}, \\
& \mathbf{H}^{i+1} \succeq 0,
\end{aligned}
$$

where $0<\lambda<1$ is the parameter controlling the trade-off between the rank of the obtained solution matrix and the stop band energy of the filter. In design examples, $\lambda$ is chosen as a function of $R^{i}$ given in (29) for improved convergence. Since obtaining a rank-1 solution is the priority in the solution of (30), $\lambda\left(R^{i}\right)$ is chosen to be a monotonic decreasing function of $R^{i}$ such as $\lambda\left(R^{i}\right)=1-\left(R^{i}\right)^{\alpha}$, where $\alpha$ is a non-negative real number that is determined by the user. For $\alpha \gg 1$, the DIRR for (30) tends to converge to a rank-1 solution faster. On the other hand, for $\alpha \approx 1$ the corresponding DIRR for (30) tends to converge to a rank-1 solution slower, but with significantly suppressed stop band energy. Choosing the appropriate values for $\alpha$ will be investigated in the next section over a set of design examples.

The total stop band energy minimization objective of the SDP given in (30) can be changed to peak stop band ripple minimization to obtain the classical low-pass FIR filter design problem [14] by replacing the semidefinite term $\operatorname{Tr}\left(\mathbf{A}_{s} \mathbf{H}^{i+1}\right)$ with a variable $\delta^{2}$ and by constraining the energy corresponding to each frequency in the stop band by $\delta^{2}$. Here, $\delta^{2}$ denotes the squared maximum stop band power allowed. The obtained SDP is given as:

$$
\begin{aligned}
& \min _{\mathbf{H}^{i+1} \in \mathbb{R}^{L \times L}, \delta^{2} \in \mathbb{R}}(1-\lambda) \delta^{2}-\lambda \operatorname{Tr}\left(\mathbf{u}_{\ell}^{i} \mathbf{u}_{\ell}^{i T} \mathbf{H}^{i+1}\right) \\
& \text { s.t. } \operatorname{Tr}\left(\mathbf{A}_{k} \mathbf{H}^{i+1}\right) \leq a_{k}, \quad 0 \leq k \leq K_{s}, \\
& \operatorname{Tr}\left(\mathbf{A}_{k} \mathbf{H}^{i+1}\right) \leq \delta^{2}, \quad K_{s}<k \leq K_{u}, \\
& \operatorname{Tr}\left(\mathbf{A}_{k} \mathbf{H}^{i+1}\right) \geq b_{k}, \quad 0 \leq k \leq K_{l}, \\
& \mathbf{H}^{i+1} \succeq 0,
\end{aligned}
$$

where $K_{s}=\left\lceil K \omega_{s} / \pi\right\rceil$. Since the objective and the newly introduced constraints are convex, (31) is still an iterative convex SDP problem. Hence, (31) can be solved by using the DIRR by choosing (31) at steps 2 and 4 in Algorithm 1 and Algorithm 2, respectively. 


\section{B. Design With Magnitude and Phase Response Constraints}

Due to their constant group delay properties, the linear phase filters are commonly used in practice. However, linear phase response requires the filter coefficients to have a certain symmetry, resulting in reduced degrees of freedom in the corresponding design. Therefore, longer filters are used to satisfy the magnitude constraints. Hence, there is a tradeoff between the magnitude response and the phase response linearity of the filter. To operate at a desired point in this tradeoff, additional constraints on the phase response can be introduced on the constructed optimization problem. An exchange based method is proposed in [7] for handling these type of constraints. However, since the constructed problem is non-convex, substantial effort is required for the convergence of the algorithm. An approach based on least squares and SDP is proposed in [40], where a desired filter response is used as a template. Unlike these approaches, here, the spectral mask constraints on the phase response of the filter are imposed in the proposed convex formulation.

Consider the following constraints on the phase response $\Phi(\omega)=\measuredangle H(\omega)$ of the filter:

$$
\left|\Phi\left(\omega_{k}\right)\right| \leq \Phi_{p}\left(\omega_{k}\right), 1 \leq k \leq K_{p},
$$

where $\omega_{k} \in[0, \pi / 2]$ for $1 \leq k \leq K_{p}$. These constraints can be related to the real and imaginary components of $H\left(\omega_{k}\right)$ as

$$
-\Phi_{p}\left(\omega_{k}\right) \leq \tan ^{-1}\left(\frac{\operatorname{Im}\left(H\left(\omega_{k}\right)\right)}{\operatorname{Re}\left(H\left(\omega_{k}\right)\right)}\right) \leq \Phi_{p}\left(\omega_{k}\right) .
$$

Since $\tan (\omega)$ is a monotonically increasing function and continuous in the interval $\omega \in[0, \pi / 2]$, (33) can be equivalently written as

$$
-\tan \left(\Phi_{p}\left(\omega_{k}\right)\right) \leq \frac{\operatorname{Im}\left(H\left(\omega_{k}\right)\right)}{\operatorname{Re}\left(H\left(\omega_{k}\right)\right)} \leq \tan \left(\Phi_{p}(\omega)\right) .
$$

Then, the squared magnitudes yields the following inequality:

$$
\left|\operatorname{Im}\left(H\left(\omega_{k}\right)\right)\right|^{2}-\left|\operatorname{Re}\left(H\left(\omega_{k}\right)\right)\right|^{2} \beta\left(\omega_{k}\right) \leq 0,
$$

where $\beta\left(\omega_{k}\right)=\tan ^{2}\left(\Phi_{p}\left(\omega_{k}\right)\right)$. The following vectors help simplifying the notation:

$$
\begin{aligned}
& \mathbf{s}\left(\omega_{k}\right)=\left[1,-\sin \left(\omega_{k}\right), \ldots,-\sin \left((L-1) \omega_{k}\right)\right]^{T}, \\
& \mathbf{c}\left(\omega_{k}\right)=\left[1, \cos \left(\omega_{k}\right), \ldots, \cos \left((L-1) \omega_{k}\right)\right]^{T} .
\end{aligned}
$$

Also let $\mathbf{S}\left(\omega_{k}\right)=\mathbf{s}\left(\omega_{k}\right) \mathbf{s}\left(\omega_{k}\right)^{T}, \mathbf{C}\left(\omega_{k}\right)=\mathbf{c}\left(\omega_{k}\right) \mathbf{c}\left(\omega_{k}\right)^{T}$, and express the phase constraints as:

$$
\operatorname{Tr}\left(\mathbf{P}_{k} \mathbf{H}\right) \leq 0, \quad 1 \leq k \leq K_{p},
$$

where $\mathbf{P}_{k}=\mathbf{S}\left(\omega_{k}\right)-\beta\left(\omega_{k}\right) \mathbf{C}\left(\omega_{k}\right)$, and $\mathbf{H}=\mathbf{h} \mathbf{h}^{T}$. The corresponding convex SDP, which includes the constraints on both the magnitude and the phase responses of the filter can be expressed as:

$$
\begin{aligned}
\min _{\mathbf{H} \in \mathbb{R}^{L \times L}} & \operatorname{Tr}\left(\mathbf{A}_{s} \mathbf{H}\right) \\
\text { s.t. } & \operatorname{Tr}\left(\mathbf{A}_{k} \mathbf{H}\right) \leq a_{k}, \quad 0 \leq k \leq K_{u}, \\
& \operatorname{Tr}\left(\mathbf{A}_{k} \mathbf{H}\right) \geq b_{k}, \quad 0 \leq k \leq K_{l} \\
& \operatorname{Tr}\left(\mathbf{P}_{k} \mathbf{H}\right) \leq 0, \quad 1 \leq k \leq K_{p} \\
& \mathbf{H} \succeq 0 .
\end{aligned}
$$

In order to use the DIRR technique, (39) can be rewritten in the form of (30) as:

$$
\begin{aligned}
\min _{\mathbf{H}^{i+1} \in \mathbb{R}^{L \times L}} & \operatorname{Tr}\left(\left((1-\lambda) \mathbf{A}_{s}-\lambda \mathbf{u}_{\ell}^{i} \mathbf{u}_{\ell}^{i}\right) \mathbf{H}^{i+1}\right) \\
\text { s.t. } & \operatorname{Tr}\left(\mathbf{A}_{k} \mathbf{H}^{i+1}\right) \leq a_{k}, 0 \leq k \leq K_{u}, \\
& \operatorname{Tr}\left(\mathbf{A}_{k} \mathbf{H}^{i+1}\right) \geq b_{k}, 0 \leq k \leq K_{l}, \\
& \operatorname{Tr}\left(\mathbf{P}_{k} \mathbf{H}^{i+1}\right) \leq 0,1 \leq k \leq K_{p}, \\
& \mathbf{H}^{i+1} \succeq 0 .
\end{aligned}
$$

\section{Design With Magnitude Response and Group Delay Constraints}

Similar to the incorporation of phase constraints, group delay constraints can also be imposed on the filter design. The following group delay mask constraints can be imposed in the optimization:

$$
G_{l}\left(\omega_{k}\right) \leq G\left(\omega_{k}\right) \leq G_{u}\left(\omega_{k}\right), 0 \leq k \leq K_{g}
$$

where $G_{l}\left(\omega_{k}\right)$ and $G_{u}\left(\omega_{k}\right)$ are the lower and the upper group delay constraints on the group delay $G\left(\omega_{k}\right)$ defined as:

$$
G\left(\omega_{k}\right)=-\left.\frac{d \Phi(\omega)}{d \omega}\right|_{\omega=\omega_{k}}
$$

By using the following derivative identity for $\tan ^{-1}(\cdot)$

$$
\frac{d \tan ^{-1} f(\omega)}{d \omega}=\frac{1}{1+f^{2}(\omega)} \frac{d f(\omega)}{d \omega},
$$

the group delay can be written as:

$$
G\left(\omega_{k}\right)=-\left.\frac{1}{1+\frac{\operatorname{Im}^{2}\left(H\left(\omega_{k}\right)\right)}{\operatorname{Re}^{2}\left(H\left(\omega_{k}\right)\right)}} \frac{d\left(\frac{\operatorname{Im}(H(\omega))}{\operatorname{Re}(H(\omega))}\right)}{d \omega}\right|_{\omega=\omega_{k}} .
$$

Now, by using (36) and (37) we obtain:

$$
G\left(\omega_{k}\right)=-\left.\frac{1}{1+\frac{\left|\mathbf{s}\left(\omega_{k}\right)^{T} \mathbf{h}\right|^{2}}{\left|\mathbf{c}\left(\omega_{k}\right)^{T} \mathbf{h}\right|^{2}}} \frac{d\left(\frac{\mathbf{s}(\omega)^{T} \mathbf{h}}{\mathbf{c}(\omega)^{T} \mathbf{h}}\right)}{d \omega}\right|_{\omega=\omega_{k}} .
$$

By using the closed form expressions of the related involved derivatives, we obtain:

$$
\begin{aligned}
G\left(\omega_{k}\right) & =\frac{\left(\mathbf{s}\left(\omega_{k}\right)^{T} \mathbf{h}\right)^{T} \tilde{\mathbf{c}}\left(\omega_{k}\right)^{T} \mathbf{h}-\left(\mathbf{c}\left(\omega_{k}\right)^{T} \mathbf{h}\right)^{T} \tilde{\mathbf{s}}\left(\omega_{k}\right)^{T} \mathbf{h}}{\mathbf{h}^{T} \mathbf{A}\left(\omega_{k}\right) \mathbf{h}} \\
& =\frac{\mathbf{h}^{T}\left(\mathbf{s}_{k} \tilde{\mathbf{c}}_{k}^{T}-\mathbf{c}_{k} \tilde{\mathbf{s}}_{k}^{T}\right) \mathbf{h}}{\mathbf{h}^{T} \mathbf{A}_{k} \mathbf{h}}
\end{aligned}
$$

where $\tilde{\mathbf{c}}(\omega)$ and $\tilde{\mathbf{s}}(\omega)$ are defined as:

$$
\begin{aligned}
& \tilde{\mathbf{c}}\left(\omega_{k}\right)=\left[0,-\sin \omega_{k}, \ldots,-(L-1) \sin (L-1) \omega_{k}\right]^{T}, \\
& \tilde{\mathbf{s}}\left(\omega_{k}\right)=\left[0,-\cos \omega_{k}, \ldots,-(L-1) \cos (L-1) \omega_{k}\right]^{T},
\end{aligned}
$$

which leads to the following form:

$$
G\left(\omega_{k}\right)=\frac{\operatorname{Tr}\left(\mathbf{D}_{k} \mathbf{H}\right)}{\operatorname{Tr}\left(\mathbf{A}_{k} \mathbf{H}\right)}
$$


where $\mathbf{D}_{k}=\mathbf{s}_{k} \tilde{\mathbf{c}}_{k}^{T}-\mathbf{c}_{k} \tilde{\mathbf{s}}_{k}^{T}$ and $\mathbf{H}=\mathbf{h h}^{T}$. Using this compact expression, the group delay constraints in (41) can be expressed as:

$$
\operatorname{Tr}\left(\mathbf{A}_{k} \mathbf{H}\right) G_{l}\left(\omega_{k}\right) \leq \operatorname{Tr}\left(\mathbf{D}_{k} \mathbf{H}\right) \leq \operatorname{Tr}\left(\mathbf{A}_{k} \mathbf{H}\right) G_{u}\left(\omega_{k}\right),
$$

for $0 \leq k \leq K_{g}$. Finally (50) can be separated into two sets of constraints as:

$$
\begin{aligned}
& \operatorname{Tr}\left(\mathbf{L}_{k} \mathbf{H}\right) \leq 0, \quad 0 \leq k \leq K_{g}, \\
& \operatorname{Tr}\left(\mathbf{U}_{k} \mathbf{H}\right) \geq 0, \quad 0 \leq k \leq K_{g},
\end{aligned}
$$

where $\mathbf{L}_{k}=G_{l}\left(\omega_{k}\right) \mathbf{A}_{k}-\mathbf{D}_{k}$ and $\mathbf{U}_{k}=G_{u}\left(\omega_{k}\right) \mathbf{A}_{k}-\mathbf{D}_{k}$. The corresponding convex SDP can be expressed as:

$$
\begin{aligned}
\min _{\mathbf{H} \in \mathbb{R}^{L \times L}} & \operatorname{Tr}\left(\mathbf{A}_{s} \mathbf{H}\right) \\
\text { s.t. } & \operatorname{Tr}\left(\mathbf{A}_{k} \mathbf{H}\right) \leq a_{k}, 0 \leq k \leq K_{u}, \\
& \operatorname{Tr}\left(\mathbf{A}_{k} \mathbf{H}\right) \geq b_{k}, 0 \leq k \leq K_{l}, \\
& \operatorname{Tr}\left(\mathbf{L}_{k} \mathbf{H}\right) \leq 0,0 \leq k \leq K_{g}, \\
& \operatorname{Tr}\left(\mathbf{U}_{k} \mathbf{H}\right) \geq 0,0 \leq k \leq K_{g}, \\
& \mathbf{H} \succeq 0 .
\end{aligned}
$$

Though (53) is a relaxed convex SDP, it does not guarantee a rank-1 solution matrix $\mathbf{H}$. By combining the formulations (30) and (53), an iterative formulation that can produce low-rank solution matrices for the group delay constrained FIR filter design is obtained as:

$$
\begin{aligned}
\min _{\mathbf{H}^{i+1} \in \mathbb{R}^{L \times L}} & \operatorname{Tr}\left(\left((1-\lambda) \mathbf{A}_{s}-\lambda \mathbf{u}_{\ell}^{i} \mathbf{u}_{\ell}^{i T}\right) \mathbf{H}^{i+1}\right) \\
\text { s.t. } & \operatorname{Tr}\left(\mathbf{A}_{k} \mathbf{H}^{i+1}\right) \leq a_{k}, 0 \leq k \leq K_{u}, \\
& \operatorname{Tr}\left(\mathbf{A}_{k} \mathbf{H}^{i+1}\right) \geq b_{k}, 0 \leq k \leq K_{l}, \\
& \operatorname{Tr}\left(\mathbf{L}_{k} \mathbf{H}^{i+1}\right) \leq 0,0 \leq k \leq K_{g}, \\
& \operatorname{Tr}\left(\mathbf{U}_{k} \mathbf{H}^{i+1}\right) \geq 0,0 \leq k \leq K_{g}, \\
& \mathbf{H}^{i+1} \succeq 0 .
\end{aligned}
$$

On this final form, the proposed iterative technique can be used. Note that, the development that led to (54) enabled the exact implementation of the group delay constraints under the desired optimization framework. An alternative technique, where the least squares error from a desired group delay response is expressed in terms of the LMI constraints under an SDP framework, is presented in [41]. However, since the corresponding LMI matrix is not guaranteed to be PSD, the use of an approximation of the LMI matrix might become necessary. Then, the constructed optimization problem will be using the approximate implementation of the original group delay constraints. Hence, unlike the existing least squares based techniques, which obtain approximations to the desired group delay responses, the proposed technique is capable of designing FIR filters with the incorporation of the actual group delay mask constraints.

\section{Design EXAMPles}

In this section, a set of illustrative FIR filter designs are presented by using the proposed DIRR technique. The obtained results are compared with the results of alternative design techniques where available. Note that, in the presented examples,

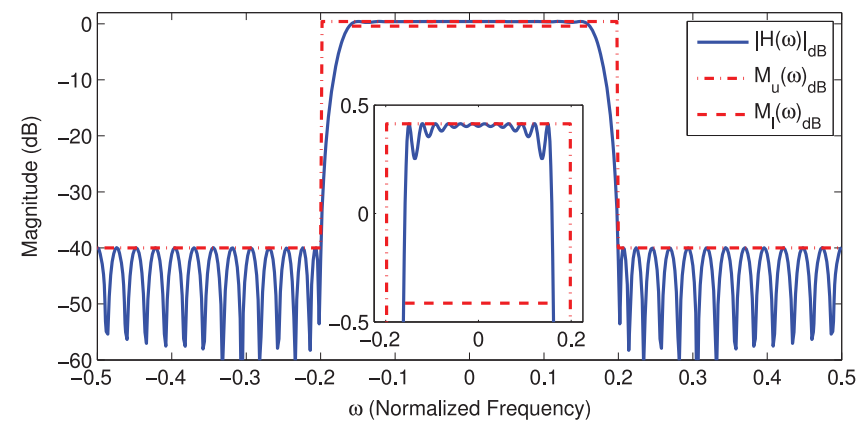

Fig. 2. Magnitude response of the FIR filter of length $L=38$ obtained in Design 1 by using the DIRR technique. The magnitude response satisfies the constraints of Design 1 with a stop band energy of $-42.9 \mathrm{~dB}$.

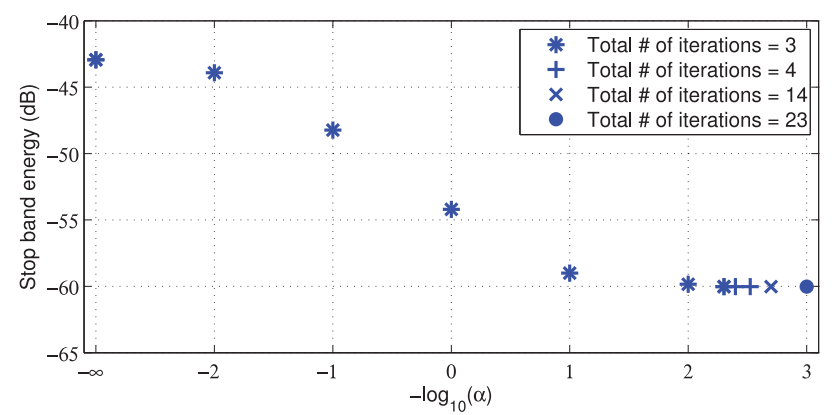

Fig. 3. As a function of $\alpha$ defined after (30) and number of DIRR iterations, the corresponding stop band energy of the designed filter. The appropriate value of $\alpha$ that determines the design parameter $\lambda$ in Alg. 2 can be identified using this plot. The largest value of $-\log _{10}(\alpha)$ for the smallest total number of the DIRR iterations provides robust designs.

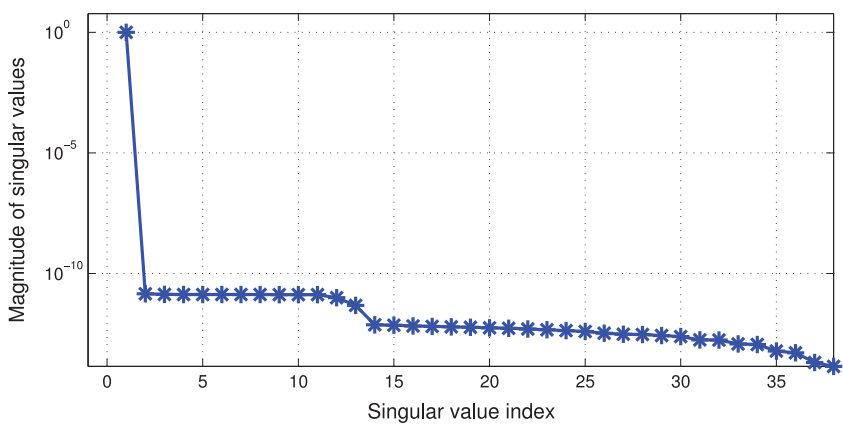

Fig. 4. The singular values of $\mathbf{H}^{i}$ obtained at the 3rd DIRR iteration for the feasibility problem given in Design 1.

the semidefinite programming solver, SDPT3, of the convex optimization tool CVX [27], [42] is used to obtain solutions to the relaxed SDP at its best resolution option.

Design 1: For the lower and upper magnitude response mask constraints, $M_{l}(\omega)$ and $M_{u}(\omega)$ shown in Fig. 2, design a filter of length $L=38$.

Since Design 1 is a feasibility problem, the optimization problem in (30) is solved by using the DIRR technique with the choice of $\ell=1$ and $\lambda=1$, which is the $\alpha=\infty$ case (the leftmost point indicated by asterisk) in Fig. 3. The corresponding singular values of the obtained solution matrix are shown in Fig. 4. Since the first singular value significantly dominates the rest, the obtained solution matrix $\mathbf{H}$ is practically rank-1. As observed in Fig. 2, the magnitude response of the designed filter satisfies all the spectral mask constraints. 


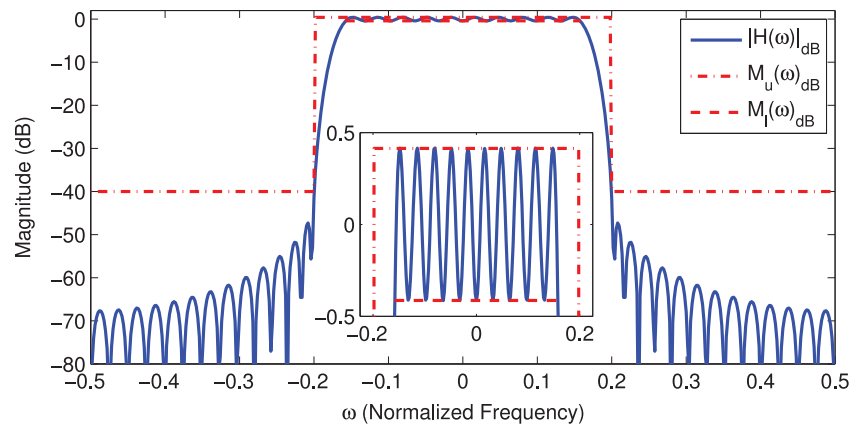

Fig. 5. Magnitude response of the FIR filter of length 38 obtained in Design 2 by using the DIRR technique. The magnitude response satisfies the constraints of Design 2 with a stop band energy of $-60.0 \mathrm{~dB}$.

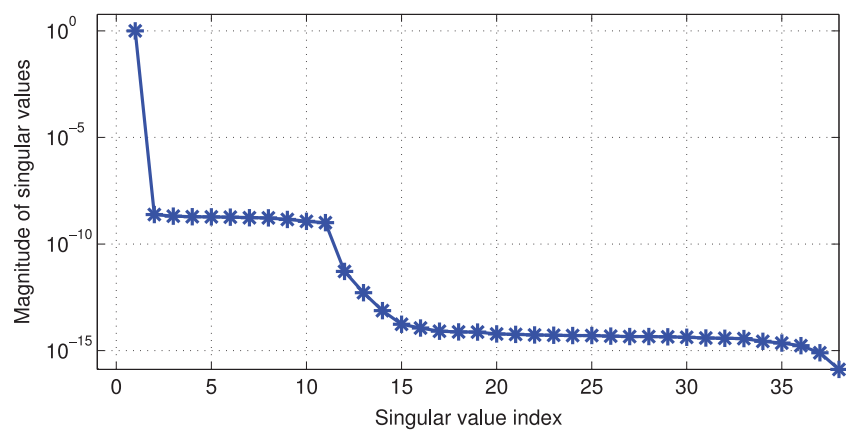

Fig. 6. The singular values of $\mathbf{H}^{i}$ at the 3rd DIRR iteration in Design 2.

Design 2: For the lower and upper magnitude response mask constraints, $M_{l}(\omega)$ and $M_{u}(\omega)$ shown in Fig. 5, design a filter of length $L=38$ with the minimum stop band energy.

In this design, the optimization problem given in (30) is solved by using the DIRR with $\ell=1$ and $\lambda=1-\left(R^{i}\right)^{\alpha}$ for $\alpha=0.005$, where $R^{i}$ is defined in (29). The exponent $\alpha$ on $R^{i}$ can be chosen even smaller to increase the emphasis on the goal of stop band energy minimization. However, with this choice of $\lambda$, the proposed DIRR technique is able to converge in only 3 iterations, with a stop band energy of $-60.0 \mathrm{~dB}$. As shown in Fig. 5, the magnitude response of the designed filter satisfies all the spectral mask constraints. As seen from Fig. 6, the obtained solution matrix at the 3 rd iteration is rank-1 for all practical purposes.

For comparison purposes, we also provide the design by the autocorrelation based design technique in [12]. Since there is no phase constraints, the autocorrelation based design technique provides the global optimal for this design. The magnitude response of the resulting filter coefficients provided by this technique is shown in Fig. 7 with a stop band energy of $-60.0 \mathrm{~dB}$. Note that, the proposed DIRR technique provides a filter with almost identical performance with the global optimal filter provided by the autocorrelation based design technique.

Design 3: For the lower and upper magnitude response mask constraints, $M_{l}(\omega)$ and $M_{u}(\omega), 0 \leq \omega \leq \omega_{s}$ shown in Fig. 8, design a filter of length $L=38$ with the minimum peak stop band ripple.

In this design, the optimization problem given in (31) is solved by using the DIRR technique with $\ell=1$ and $\lambda=1-\left(R^{i}\right)^{\alpha}$ for $\alpha=10^{-5}$. The proposed DIRR technique converged in 24 iterations, with a stop band energy of

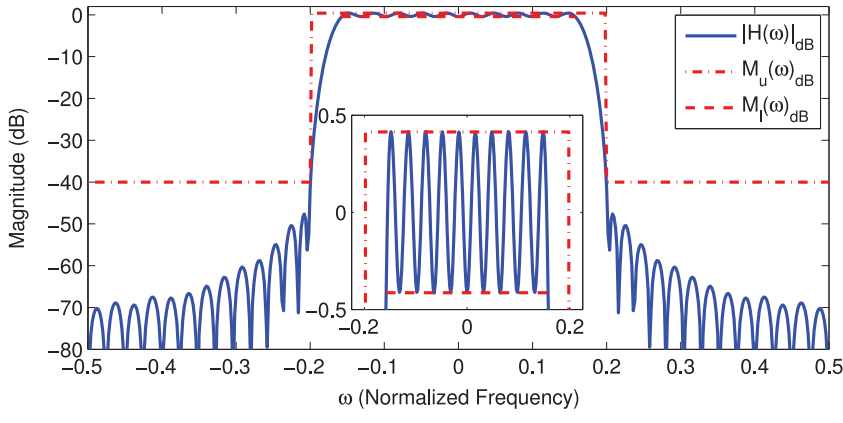

Fig. 7. Magnitude response of the FIR filter of length 38 obtained in Design 2 by the autocorrelation based LP technique in [12]. The magnitude response satisfies the constraints of Design 2 with a stop band energy of $-60.0 \mathrm{~dB}$.

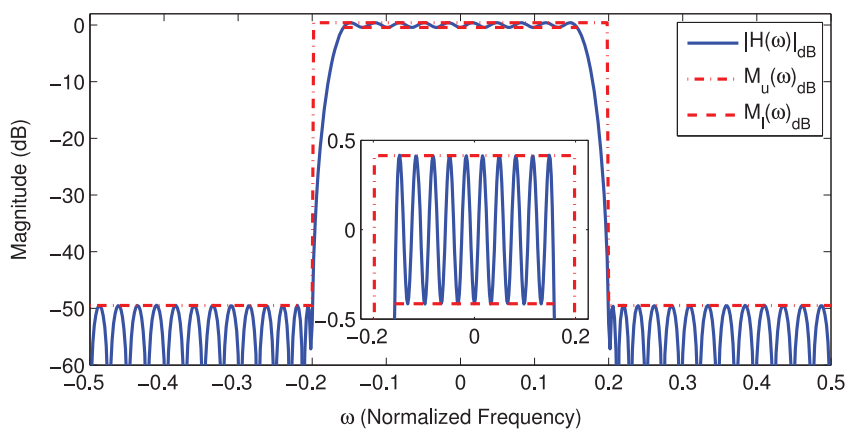

Fig. 8. Magnitude response of the FIR filter of length 38 obtained in Design 3 using the DIRR technique. The total stop band energy is $-52.5 \mathrm{~dB}$ and the peak stop band ripple is $\delta^{2}=-49.5 \mathrm{~dB}$.

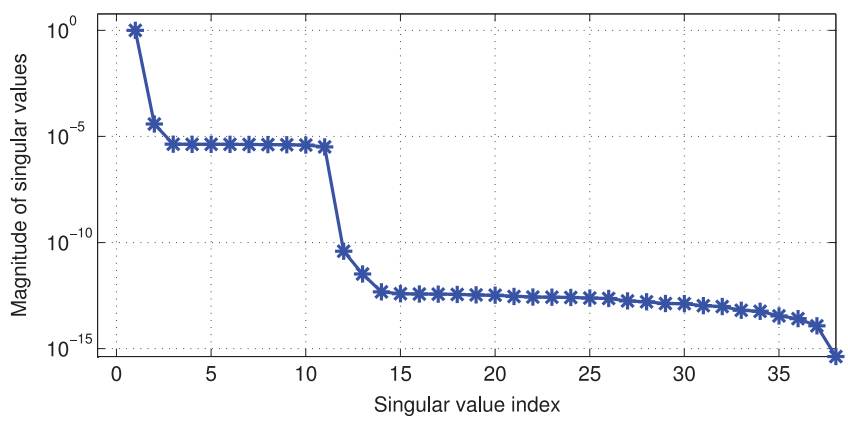

Fig. 9. The singular values of $\mathbf{H}^{i}$ at the 24th DIRR iteration in Design 3.

$-52.5 \mathrm{~dB}$. As shown in Fig. 9, the ratio of the first singular value to the sum of all singular values is $R^{i}=0.99992$ at iteration $i=24$, indicating that the obtained solution matrix is practically a rank-1 matrix. As shown in Fig. 8, the designed filter satisfies the constraints with a peak stop band ripple of $\delta^{2}=-49.5 \mathrm{~dB}$. Since there are no constraints on the phase response of the filter in this design, the autocorrelation based LP technique [12] provides the global optimal design shown in Fig. 10 with a stop band energy of $-52.6 \mathrm{~dB}$ and peak stop band ripple of $\delta^{2}=-49.6 \mathrm{~dB}$. Note that the DIRR technique provides almost identical performance.

Design 4: For the magnitude and phase response constraints, $M_{l}(\omega), M_{u}(\omega)$ shown in Fig. 11 and $\Phi_{p}(\omega)$ shown in Fig. 12, respectively, design the lowest order filter with the minimum stop band energy.

Constraints on the phase and group delay responses normally cannot be implemented exactly in techniques that utilize convex 


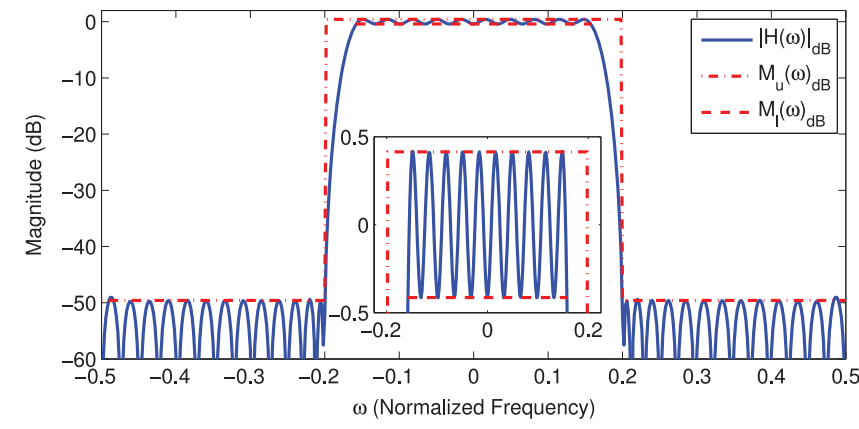

Fig. 10. Magnitude response of the FIR filter of length 38 obtained in Design 3 using the autocorrelation based LP technique. The total stop band energy is $-52.6 \mathrm{~dB}$ and the peak stop band ripple is $\delta^{2}=-49.6 \mathrm{~dB}$.

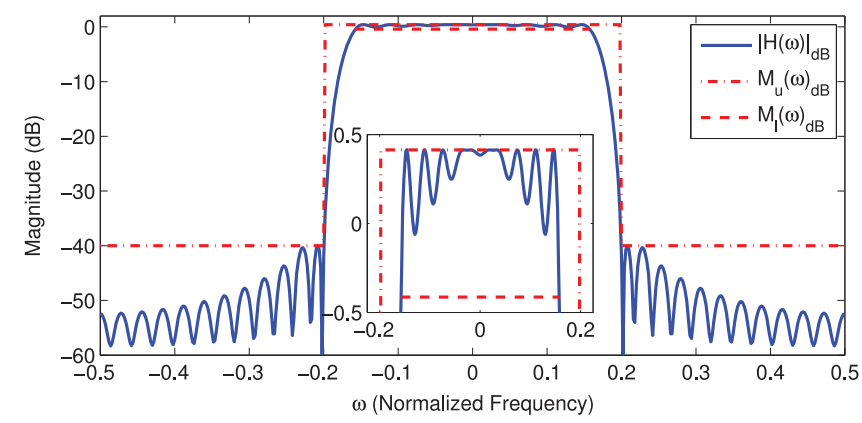

Fig. 11. Magnitude response of the FIR filter of length 35 obtained in Design 4 using the DIRR technique. The magnitude response satisfies the constraints of Design 4 with a stop band energy of $-48.8 \mathrm{~dB}$.

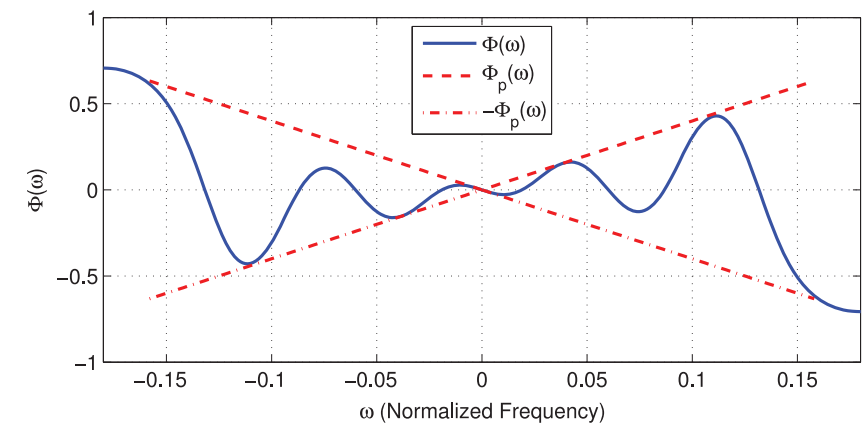

Fig. 12. Phase response of the FIR filter of length 35 obtained in Design 4 using the DIRR technique. The upper and the lower phase mask constraints that are satisfied by the designed filter are given as $\Phi_{p}(\omega)=4|\omega|+4 \times 10^{-5}$ for $|\omega|<0.16$.

optimization such as the autocorrelation based LP technique that have provided the optimal results in the previous design examples. Even though minimax type of optimization formulations can implement such constraints, they do not provide freedom for the user to choose the stop band energy minimization as an objective [6], [7], [15]. However, since the proposed DIRR technique is capable of exactly incorporating these constraints in the SDP form, the phase and the group delay constraints can be handled together with the energy minimization objectives.

In order to obtain the FIR filter with the lowest order satisfying the given constraints, (40) can be solved for a range of filter orders. In this design, the DIRR technique with parameters $\lambda=1-\left(R^{i}\right)^{\alpha}$ for $\alpha=1$ is used to obtain the solution which is on the direction of the 2 nd singular vector of $\mathbf{H}^{1}$ at the 3 rd iteration with $R^{i}=0.99991$. The corresponding singular values are

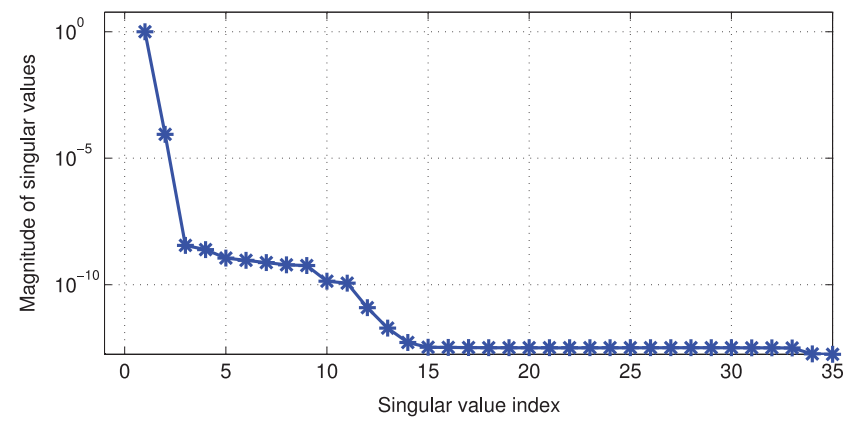

Fig. 13. Singular values of $\mathbf{H}^{i}$ at the 3rd DIRR iteration in Design 4.

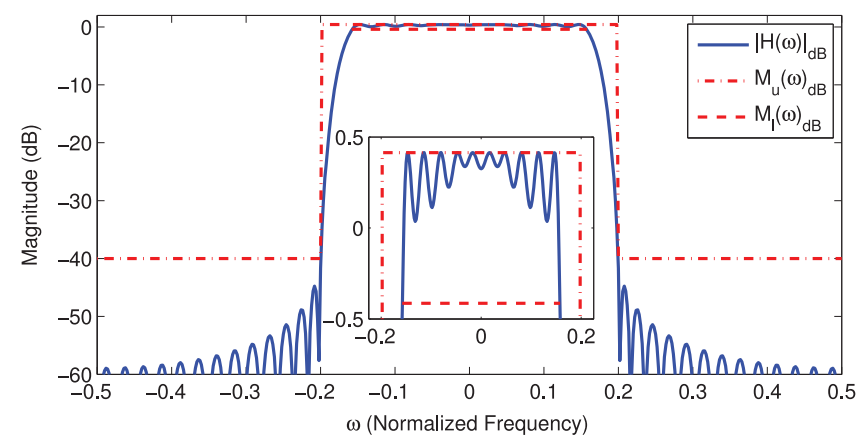

Fig. 14. Magnitude response of the FIR filter of length 38 obtained in Design 5 by using the DIRR technique. The magnitude response satisfies constraints of Design 5 with a stop band energy of $-56.3 \mathrm{~dB}$.

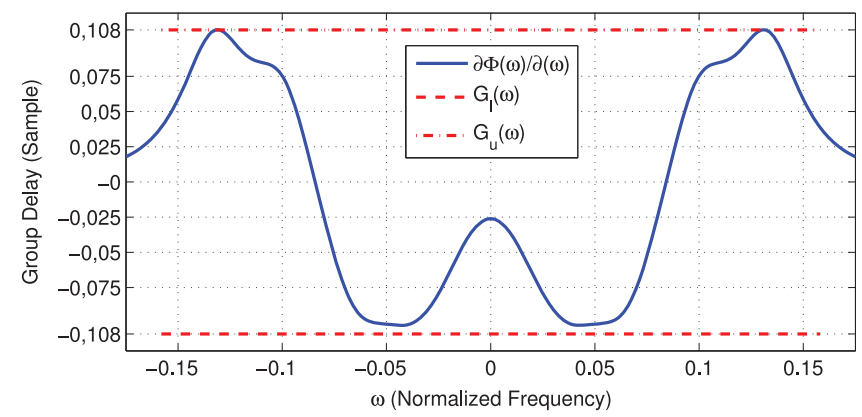

Fig. 15. Group delay of the FIR filter of length 38 obtained in Design 5 by using the DIRR technique. The group delay of the designed filter satisfies the constraints of Design 5, which are $G_{u}(\omega)=-G_{l}(\omega)=0.108$ for $|\omega|<$ 0.16 .

shown in Fig. 13. The resulting filter order is found to be 35. As shown in Figs. 11 and 12, all the constraints on the magnitude and phase responses are satisfied by the designed filter which has a stop band energy of $-48.8 \mathrm{~dB}$. The LP based linear phase filter design technique proposed in [12] required a filter order of 39 to meet the constraints of this design.

Design 5: For the magnitude constraints, $M_{l}(\omega)$ and $M_{u}(\omega)$ shown in Fig. 14, and the group delay mask constraints $G_{l}\left(\omega_{k}\right)$ and $G_{u}\left(\omega_{k}\right)$ shown in Fig. 15, find the filter of the lowest order with the minimum stop band energy.

In this design, the optimization problem in (54) is solved by using the DIRR technique with $\lambda=1-\left(R^{i}\right)^{\alpha}$ for $\alpha=1$. The proposed DIRR technique has converged to a solution in 4 iterations on the direction of the 6th singular vector with the singular value ratio of 1 . The resulting filter order is found to be 38 . The magnitude and group delay responses of the designed filter are 


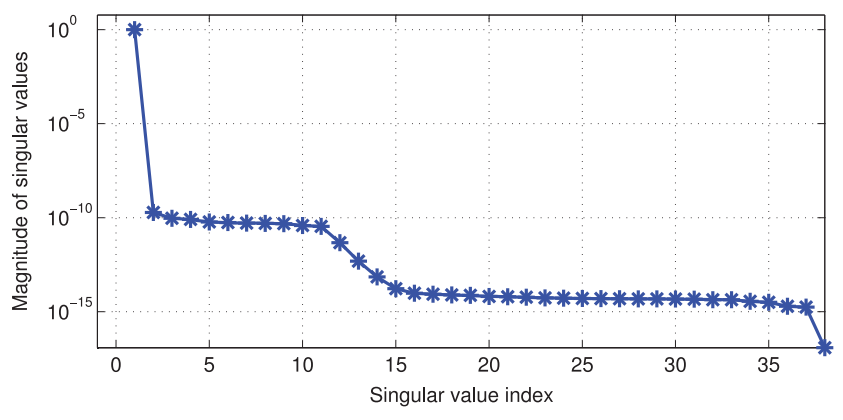

Fig. 16. Singular values of $\mathbf{H}^{i}$ at the 4th DIRR iteration in Design 5.

TABLE I

THE COMPARISON OF MINIMUM FILTER ORDERS FOR DIFFERENT DIRR Formulations, Optimal NONLINEAR AND LINEAR Phase Formulations GIVEN IN [12]. $1: \omega_{p}=0.16, \omega_{s}=0.20 ; 2: \omega_{p}=0.16, \omega_{s}=0.24 ; 3$ : $\omega_{p}=0.16, \omega_{s}=0.22 ; 4: \omega_{p}=0.16, \omega_{s}=0.19 ; 5: \omega_{p}=0.10, \omega_{s}=0.14$

\begin{tabular}{|c|c|c|c|c|c|c|}
\hline & $\begin{array}{c}\text { [12] Nonlin. } \\
\text { Phase }\end{array}$ & $(30)$ & $\begin{array}{c}\text { (40) in } \\
\text { Fig. 12 }\end{array}$ & $\begin{array}{c}\text { (54) in } \\
\text { Fig. 15 }\end{array}$ & $\begin{array}{c}\text { [12] Lin. } \\
\text { Phase }\end{array}$ & $\begin{array}{c}\text { (40) Lin. } \\
\text { Phase }\end{array}$ \\
\hline 1 & 32 & 32 & 35 & 38 & 39 & 39 \\
2 & 17 & 17 & 19 & 17 & 20 & 20 \\
3 & 23 & 23 & 25 & 23 & 27 & 27 \\
4 & 33 & 33 & 39 & 33 & 40 & 40 \\
5 & 41 & 41 & 45 & 41 & 50 & 50 \\
\hline
\end{tabular}

shown in Figs. 14 and 15, respectively. The stop band energy is $-56.3 \mathrm{~dB}$. As observed, all the constraints on the magnitude and group delay responses of the filter are satisfied. The resulting singular values, shown in Fig. 16, demonstrate the dominance of the first singular value over the rest.

Finally, in Table I, we have compared the order of the filters designed by the proposed formulations in (30), (40) and (54) with the linear and non-linear phase response filter design methods in [12]. In the comparisons, we have used the spectral mask constraints given in Fig. 1 for various values of pass band and stop band corner frequencies $\left(\omega_{p}\right.$ and $\left.\omega_{s}\right)$. The phase constraints in Fig. 12 and group delay constraints in Fig. 15 have been used for the designs by (40) and (54), respectively. We have also provided a linear phase response design using (40) by simply setting $\Phi_{p}(w)=0 \forall|w| \leq \omega_{p}$. For the linear and nonlinear phase filter design cases, the results suggest that DIRR achieves the same filter order provided by the optimal method given in [12]. Moreover, DIRR formulations in (40) and (54) provide lower filter order solutions compared to the optimal linear phase response filter design method in [12], which is the main advantage of DIRR over the existing mask constrained techniques.

\section{CONCLUSION}

Recent advances in the optimization techniques have provided an opportunity to incorporate the phase and group delay constraints in the optimization based formulation of the filter design problem. However, the straightforward formulation of such designs results in non-convex optimization problems in terms of the impulse response $\mathbf{h}$. By using the lifting techniques, where the variable of optimization is mapped to a rank-1 matrix $\mathbf{H}=\mathbf{h h}^{T}$, the non-convex problem can be converted to a convex problem on $\mathbf{H}$ if we ignore the rank-1 constraint on $\mathbf{H}$ [24], [33], [35]. However, the relaxation of the rank-1 constraint results in significant degradation on the obtained results. To alleviate this issue of the relaxed convex formulation, here, an iterative optimization technique is proposed. In the DIRR technique, a sequence of convex optimization problems are solved to minimize an adaptively chosen cost function which steers the solution matrix towards a rank- 1 solution. It is proven that the global optimal solution to the filter design problem is a fixed point of the proposed iterations. The design performance of the proposed DIRR technique is extensively illustrated over design cases under a variety of constraints. The obtained results and comparisons have shown that the proposed DIRR technique provides robust designs which are significantly better than the alternative design techniques under the phase and group delay constraints.

\section{REFERENCES}

[1] T. Davidson, "Enriching the art of FIR filter design via convex optimization," IEEE Signal Process. Mag., vol. 27, no. 3, pp. 89-101, May 2010.

[2] J. McClellan and T. Parks, "A unified approach to the design of optimum FIR linear-phase digital filters," IEEE Trans. Circuit Theory, vol. CT-20, no. 6, pp. 697-701, Nov. 1973.

[3] K. Kose and A. E. Cetin, "Low-pass filtering of irregularly sampled signals using a set theoretic framework [lecture notes]," IEEE Signal Process. Mag., vol. 28, no. 4, pp. 117-121, Jul. 2011.

[4] J. McClellan and T. Parks, "A personal history of the Parks-McClellan algorithm,” IEEE Signal Process. Mag., vol. 22, no. 2, pp. 82-86, Mar. 2005.

[5] K. Steiglitz, T. Parks, and J. Kaiser, "METEOR: A constraint-based FIR filter design program," IEEE Trans. Signal Process., vol. 40, no. 8, pp. 1901-1909, Aug. 1992.

[6] J. Adams, "FIR digital filters with least-squares stopbands subject to peak-gain constraints," IEEE Trans. Circuits Syst., vol. 38, no. 4, pp. 376-388, Apr. 1991.

[7] J. Adams and J. Sullivan, "Peak-constrained least-squares optimization," IEEE Trans. Signal Process., vol. 46, no. 2, pp. 306-321, Feb. 1998.

[8] T. Parks and J. McClellan, "Chebyshev approximation for nonrecursive digital filters with linear phase," IEEE Trans. Circuit Theory, vol. 19, no. 2, pp. 189-194, Mar. 1972.

[9] G. Oetken, "A new approach for the design of digital interpolating filters," IEEE Trans. Acoust., Speech, Signal Process., vol. 27, no. 6, pp. 637-643, Dec. 1979.

[10] X. Lai and R. Zhao, "On Chebyshev design of linear-phase FIR filters with frequency inequality constraints," IEEE Trans. Circuits Syst. II, Exp. Briefs, vol. 53, no. 2, pp. 120-124, Feb. 2006.

[11] R. Zhao and X. Lai, "Chebyshev design of linear-phase FIR filters with linear equality constraints," IEEE Trans. Circuits Syst. II, Exp. Briefs, vol. 54, no. 6, pp. 494-498, Jun. 2007.

[12] S.-P. Wu, S. Boyd, and L. Vandenberghe, "FIR filter design via semidefinite programming and spectral factorization," in Proc. 35th IEEE Conf. Decision Contr., 1996, vol. 1, pp. 271-276, vol. 1.

[13] M. S. Lobo, L. Vandenberghe, S. Boyd, and H. Lebret, "Applications of second-order cone programming," Linear Algebra Appl., vol. 284, no. 13, pp. 193-228, Nov. 1998.

[14] S. P. Wu, S. Boyd, and L. Vandenberghe, "FIR filter design via spectral factorization and convex optimization," in Applied and Computational Control, Signals and Circuits, B. N. Datta, Ed. Boston, MA, USA: Birkhauser, 1999, vol. 1, pp. 215-242.

[15] Z. Lin and Y. Liu, "Design of arbitrary complex coefficient WLS FIR filters with group delay constraints," IEEE Trans. Signal Process., vol. 57 , no. 8, pp. 3274-3279, Aug. 2009.

[16] T. Baran, D. Wei, and A. Oppenheim, "Linear programming algorithms for sparse filter design," IEEE Trans. Signal Process., vol. 58, no. 3, pp. 1605-1617, Mar. 2010.

[17] X. Lai, "Optimal design of nonlinear-phase FIR filters with prescribed phase error," IEEE Trans. Signal Process., vol. 57, no. 9, pp. 3399-3410, Sep. 2009.

[18] X. Lai and Z. Lin, "Optimal design of constrained FIR filters without phase response specifications," IEEE Trans. Signal Process., vol. 62, no. 17, pp. 4532-4546, Sep. 2014. 
[19] I. Dotlic and R. Kohno, "Design of the family of orthogonal and spectrally efficient UWB waveforms," IEEE J. Sel. Topics Signal Process., vol. 1, no. 1, pp. 21-30, Jun. 2007.

[20] X. Wu, Z. Tian, T. Davidson, and G. Giannakis, "Optimal waveform design for UWB radios," IEEE Trans. Signal Process., vol. 54, no. 6, pp. 2009-2021, Jun. 2006.

[21] Y. K. Alp, M. Dedeoglu, and O. Arikan, "Ultra-wideband orthogonal pulse shape set design by using hermite-gaussian functions," in Proc. IEEE 20th Signal Process. Commun. Appl. Conf. (SIU), 2012, pp. 1-4.

[22] J. Mattingley and S. Boyd, "Real-time convex optimization in signal processing," IEEE Signal Process. Mag., vol. 27, no. 3, pp. 50-61, May 2010.

[23] T. Davidson, Z.-Q. Luo, and J. Sturm, "Linear matrix inequality formulation of spectral mask constraints with applications to FIR filter design," IEEE Trans. Signal Process., vol. 50, no. 11, pp. 2702-2715, Nov. 2002.

[24] Z.-Q. Luo, W.-K. Ma, A.-C. So, Y. Ye, and S. Zhang, "Semidefinite relaxation of quadratic optimization problems," IEEE Signal Process. Mag., vol. 27, no. 3, pp. 20-34, May 2010.

[25] J. Lofberg, "YALMIP: A toolbox for modeling and optimization in MATLAB," in Proc. IEEE Int. Symp. Comput.-Aided Contr. Syst. Design, Sept. 2004, pp. 284-289.

[26] J. F. Sturm, "Using sedumi 1.02, a MATLAB toolbox for optimization over symmetric cones," Optimiz. Methods Software, vol. 11-12, pp. 625-653, 1999.

[27] M. Grant and S. Boyd, "CVX: Matlab Software for Disciplined Convex Programming, Version 2.0 Beta” Sep. 2012 [Online]. Available: http:/ /cvxr.com/cvx

[28] J. Yen, "On nonuniform sampling of bandwidth-limited signals," IRE Trans. Circuit Theory, vol. 3, no. 4, pp. 251-257, Dec. 1956.

[29] A. Aldroubi and K. Gröchenig, "Nonuniform sampling and reconstruction in shift-invariant spaces," SIAM Rev., vol. 43, no. 4, pp. 585-620, Apr. 2001.

[30] R. Vaughan, N. Scott, and D. White, "The theory of bandpass sampling," IEEE Trans. Signal Process., vol. 39, no. 9, pp. 1973-1984, Sep. 1991.

[31] K. M. Anstreicher, "Semidefinite programming versus the reformulation-linearization technique for nonconvex quadratically constrained quadratic programming," J. Global Optimiz., vol. 43, pp. 471-484, 2009.

[32] M. Lang and J. Bamberger, "Nonlinear phase FIR filter design according to the $L_{2}$ norm with constraints for the complex error," Signal Process. vol. 38, no. 2, pp. 259-268, 1994 [Online]. Available: http:/ /www.sciencedirect.com/science/article/pii/0165168494901457, [Online]. Available:

[33] S. Boyd and L. Vandenberghe, Convex Optimization. Cambridge, U.K.: Cambridge Univ. Press, 2004.

[34] K. Anstreicher and H. Wolkowicz, "On lagrangian relaxation of quadratic matrix constraints," SIAM J. Matrix Anal. Appl., vol. 22, no. 1, pp. 41-55, 2000.

[35] J. Dattorro, Convex Optimization \& Euclidean Distance Geometry, J. Dattorro, Ed. Palo Alto, CA, USA: Meboo Publishing, 2005, ch. 4.

[36] G. Pataki, "On the rank of extreme matrices in semidefinite programs and the multiplicity of optimal eigenvalues," Math. Operations Res., vol. 23, no. 2, pp. 339-358, May 1998.

[37] M. Fazel, "Matrix Rank Minimization With Applications," Ph.D. dissertation, Dept. Electr. Eng., Stanford Univ., Stanford, CA, USA, 2002.

[38] M. Fazel, H. Hindi, and S. P. Boyd, "A rank minimization heuristic with application to minimum order system approximation," in Proc. IEEE Amer. Contr. Conf., 2001, vol. 6, pp. 4734-4739.
[39] A. M.-C. So, J. Zhang, and Y. Ye, "On approximating complex quadratic optimization problems via semidefinite programming relaxations," Math. Program., vol. 110, no. 1, pp. 93-110, Mar. 2007.

[40] W. S. Lu, "Design of nonlinear-phase FIR digital filters: A semidefinite programming approach," in Proc. 1999 IEEE Int. Symp. Circuits Syst. (ISCAS'99), Jul. 1999, vol. 3, pp. 263-266, vol. 3.

[41] Z. Lin and Y. Liu, "FIR filter design with group delay constraint using semidefinite programming," in Proc. IEEE Int. Symp. Circuits Syst. (ISCAS 2006), May 2006, pp. 2505-2508.

[42] M. Grant and S. Boyd, "Graph implementations for nonsmooth convex programs," in Recent Advances in Learning and Control, ser. Lecture Notes in Control and Information Sciences, V. Blondel, S. Boyd, and H. Kimura, Eds. Berlin, Germany: Springer-Verlag, 2008, pp. 95-110 [Online]. Available: http://stanford.edu/ boyd/graph_dcp.html

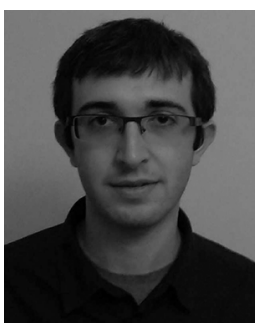

Mehmet Dedeoğlu (S'13) received both B.Sc. and M.Sc. degrees in electrical and electronics engineering from Bilkent University, Ankara, Turkey in 2012 and 2014, respectively.

$\mathrm{He}$ is currently pursuing a Ph.D. degree in electrical, computer and energy engineering at Arizona State University, Tempe, AZ. His current research is primarily focused on the study of device-to-device communications, stochastic geometry and social networks.

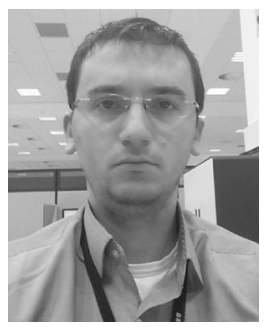

Yaşar K. Alp was born in Konya, Turkey, in 1985. He received both B.Sc. and Ph.D. degrees in electrical and electronics engineering from Bilkent University, Ankara, Turkey in 2007 and 2014, respectively.

He worked as a research scientist in Schlumberger Cambridge Research between June-August 2009 and July-September 2010. He is presently working as an algorithm design engineer in Radar, Electronic Warfare and Intelligence Systems division of ASELSAN Inc. His current research interests are time-frequency signal analysis, inverse problems and their applications on array signal processing.

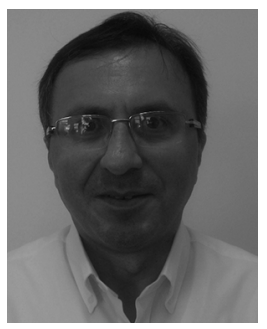

Orhan Arıkan (M'91) was born in 1964, in Manisa, Turkey. He received the B.Sc. degree in electrical and electronics engineering from the Middle East Technical University, Ankara, Turkey, in 1986, and both the M.S. and Ph.D. degrees in electrical and computer engineering from the University of Illinois, Urbana-Champaign, in 1988 and 1990, respectively.

Following his graduate studies, he worked for three years as a Research Scientist at Schlumberger-Doll Research, Ridgefield, CT. He joined Bilkent University in 1993, where he is presently Professor of Electrical Engineering since 2006 and chair of the Electrical Engineering Department since 2011. His current research interests are in statistical signal processing, time-frequency analysis, and array signal processing. 